\title{
Hiding relativistic degrees of freedom in the early universe
}

\author{
${ }^{1,7}$ V. Barger, ${ }^{2}$ James P. Kneller, ${ }^{3,7}$ Paul Langacker, ${ }^{4,7}$ Danny Marfatia and ${ }^{5,6,7}$ Gary Steigman \\ ${ }^{1}$ Department of Physics, University of Wisconsin, Madison, WI 53706 \\ ${ }^{2}$ Department of Physics, North Carolina State University, Raleigh, NC 27695 \\ ${ }^{3}$ Department of Physics and Astronomy, University of Pennsylvania, Philadelphia, PA 19104 \\ ${ }^{4}$ Department of Physics, Boston University, Boston, MA 02215 \\ ${ }^{5}$ Department of Physics, The Ohio State University, Columbus, OH 43210 \\ ${ }^{6}$ Department of Astronomy, The Ohio State University, Columbus, OH 43210 and \\ ${ }^{7}$ Kavli Institute for Theoretical Physics, University of California, Santa Barbara, CA 93106
}

\begin{abstract}
We quantify the extent to which extra relativistic energy density can be concealed by a neutrino asymmetry without conflicting with the baryon asymmetry measured by the Wilkinson Microwave Anisotropy Probe (WMAP). In the presence of a large electron neutrino asymmetry, slightly more than seven effective neutrinos are allowed by Big Bang Nucleosynthesis (BBN) and WMAP at $2 \sigma$. The same electron neutrino degeneracy that reconciles the BBN prediction for the primordial helium abundance with the observationally inferred value also reconciles the LSND neutrino with BBN by suppressing its thermalization prior to BBN.
\end{abstract}

\section{INTRODUCTION}

If the three known neutrinos $\left(\nu_{e}, \nu_{\mu}, \nu_{\tau}\right)$ mix only with each other, all active neutrino degeneracies will equilibrate to close to the electron neutrino degeneracy via neutrino oscillations before BBN begins [1]. There are no caveats to this statement within the standard cosmological model for the oscillation parameters relevant to atmospheric neutrino data and the Large Mixing Angle (LMA) solution to the solar neutrino problem (which has recently been confirmed by the KamLAND experiment [2]). Thus, the magnitude of the electron neutrino degeneracy allowed by BBN is of special interest to any determination of, or constraints on, a lepton asymmetry in the universe.

The authors of Ref. [1] emphasized that the degenerate BBN scenario, in which a small $\nu_{e}$ asymmetry ${ }^{1} \xi_{e} \sim 0.2$ (which strongly affects the neutron to proton ratio and, as a result, the primordial ${ }^{4} \mathrm{He}$ abundance) is compensated by a much larger $\nu_{\mu}$ or $\nu_{\tau}$ asymmetry $\left|\xi_{\mu, \tau}\right| \sim 2-3$ (which affects the expansion rate) is excluded by this equilibration, leading to a limit $\left|\xi_{i}\right| \lesssim 0.1$ on all of the asymmetries.

However, larger asymmetries may still be allowed if the effect of $\xi_{e}$ on BBN is compensated by a new source of energy density other than that from the asymmetries themselves ${ }^{2}$. In particular, if there are additional degrees

${ }^{1}$ For a neutrino flavor $\alpha$, an asymmetry $L_{\alpha}$ between the numbers of $\nu_{\alpha}$ and $\bar{\nu}_{\alpha}$ ("neutrino degeneracy") can be described by the neutrino chemical potential $\mu_{\alpha}$ or by the dimensionless degeneracy parameter $\xi_{\alpha} \equiv \mu_{\alpha} / T$ :

$$
L_{\alpha} \equiv \frac{n_{\nu_{\alpha}}-n_{\bar{\nu}_{\alpha}}}{n_{\gamma}}=\frac{\pi^{2}}{12 \zeta(3)}\left(\xi_{\alpha}+\frac{\xi_{\alpha}^{3}}{\pi^{2}}\right) .
$$

Note that $L_{\alpha} \approx 0.684 \xi_{\alpha}$ for $\xi_{\alpha} \ll 1$.

2 The faster expansion rate due to the new energy density could in principle also affect when or whether the equilibration occurs. of freedom which do not mix with the three active neutrinos, but which do contribute to the relativistic energy density at the BBN epoch, their presence can be hidden by an electron neutrino degeneracy. In this paper we quantify and explore the extent to which this statement is true.

As an example, light Dirac neutrinos involve three additional $S U(2)$-singlet neutrinos $\nu_{R}$, the right-handed partners of the active states. Within the standard model (extended to include the right-handed neutrinos) these states have no interactions other than those associated with neutrino mass, i.e., they can only be produced by mass effects of amplitude $m_{\nu} / E$ or the associated tiny Yukawa couplings. These are too small to yield significant number densities at the BBN epoch for masses in the sub-eV range. However, light Dirac neutrinos are especially motivated in models involving new gauge interactions beyond the standard model (which may forbid the traditional seesaw mechanism) [3]. In that case they need not be sterile with respect to the new interactions, and may be efficiently produced prior to BBN [4]. This was recently studied in detail for a class of models with $Z^{\prime}$ couplings motivated by $E_{6}$ [5]. The production and decoupling of the right-handed neutrinos, and the subsequent dilution of their number density by annihilation of decoupled heavy particles and color confinement following the quark-hadron phase transition was calculated. The results are dependent on the specific $Z^{\prime}$ couplings and mass but, typically, the energy density at BBN associated with the $\nu_{R}$ could be equivalent to that carried by 3,2 , or 1 active neutrinos for a $Z^{\prime}$ mass of $500 \mathrm{GeV}$, $1 \mathrm{TeV}$, or $2 \mathrm{TeV}$, respectively, with the constraints becoming weak or disappearing for the specific couplings for which the $Z^{\prime}$ decouples from the $\nu_{R}$.

If sterile neutrinos that mix with the active ones exist,

However, the considerations in this paper are independent of equilibration. 
whether or not flavor equilibration occurs is not known. We shall only consider this case in the context of the LSND [6] neutrino for which a large electron neutrino degeneracy suppresses its mixing with the active neutrinos [7]. There has been considerable interest in sterile neutrinos as a way to account for the antimuon neutrino to antielectron neutrino oscillation signal observed in the LSND experiment corresponding to a neutrino masssquared difference scale of about $0.1-1 \mathrm{eV}^{2}$ [8]. With three neutrinos there are only two independent masssquared difference scales, that are fixed by the observed atmospheric and solar oscillations to be about $2.5 \times 10^{-3}$ $\mathrm{eV}^{2}[9]$ and $7 \times 10^{-5} \mathrm{eV}^{2}[10]$, respectively. Consequently, oscillations to a sterile neutrino have been invoked to explain the higher mass-squared difference scale of the LSND effect. The MiniBooNE experiment [11] now running at Fermilab is designed to confirm or refute the LSND effect. Analyses with four neutrinos do not provide a good global fit to the data [12], but the possibility exists that the inclusion of certain small parameters, hitherto neglected in the analysis, may improve the fit [13].

We account for additional relativistic (at BBN) degrees of freedom " $X$ " by normalizing their contribution to the energy density to that of an equivalent, non-degenerate, neutrino flavor [14],

$$
\rho_{X} \equiv \Delta N_{\nu} \rho_{\nu}=\frac{7}{8} \Delta N_{\nu} \rho_{\gamma}
$$

The effective number of neutrinos is $N_{\nu} \equiv 3+\Delta N_{\nu}$.

Throughout this work the following primordial values are adopted for the ${ }^{4} \mathrm{He}$ mass fraction $\mathrm{Y}$ and for the deuterium abundance $y_{\mathrm{D}} \equiv 10^{5}(\mathrm{D} / \mathrm{H})$ :

$$
\mathrm{Y}=0.238 \pm 0.005, \quad y_{\mathrm{D}}=2.6 \pm 0.4
$$

For a detailed discussion of these choices, see Ref. [15].

\section{THE ROLE OF $\xi_{e}$ IN BBN}

The baryon-to-photon ratio $\eta$ provides a dimensionless measure of the universal baryon asymmetry which is very small $\left(\eta \equiv n_{\mathrm{B}} / n_{\gamma} \lesssim 10^{-9}\right)^{3}$. By charge neutrality the asymmetry in the charged leptons must also be of this order. However, there are no observational constraints, save those to be discussed here (see [16, 17] and further references therein), on the magnitude of any asymmetry among the neutral leptons (neutrinos). A relatively small asymmetry between electron type neutrinos and antineutrinos $\left(\left|\xi_{e}\right| \gtrsim 10^{-2}\right)$, but large compared to the baryon asymmetry, can have a significant impact on the early universe ratio of neutrons to protons, thereby affecting the yields of the light nuclides formed during BBN. The

\footnotetext{
${ }^{3}$ We define $\eta_{10} \equiv 10^{10} \eta$, to facilitate the use of numbers of order unity.
}

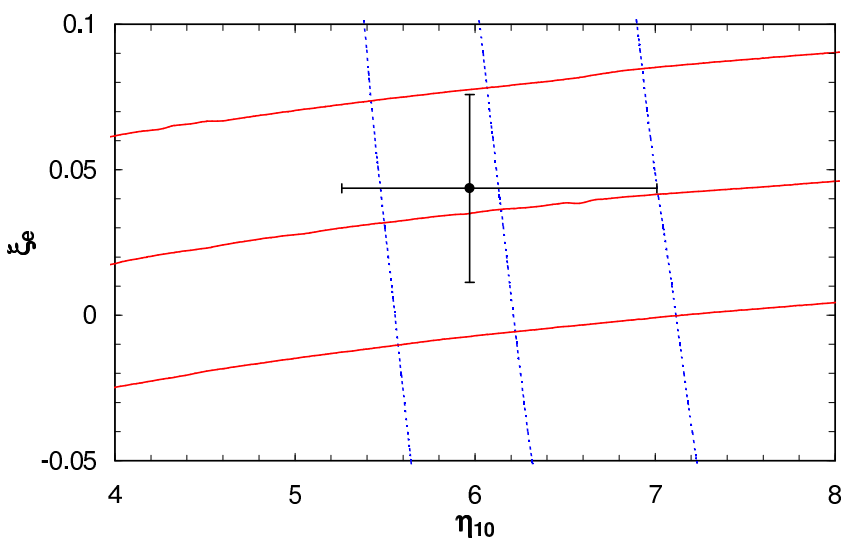

FIG. 1: Isoabundance curves for D and ${ }^{4} \mathrm{He}$ in the $\xi_{e}-\eta_{10}$ plane for $N_{\nu}=3$. The nearly horizontal curves are for ${ }^{4} \mathrm{He}$ (from top to bottom: $\mathrm{Y}=0.23,0.24,0.25$ ). The nearly vertical curves are for $\mathrm{D}$ (from left to right: $y_{\mathrm{D}} \equiv 10^{5}(\mathrm{D} / \mathrm{H})=$ $3.0,2.5,2.0)$. The data point with error bars corresponds to $y_{\mathrm{D}}=2.6 \pm 0.4$ and $\mathrm{Y}=0.238 \pm 0.005$.

strongest effect is on the ${ }^{4} \mathrm{He}$ abundance, which, during $\mathrm{BBN}$, is neutron limited. For $\xi_{e}>0$, there is an excess of neutrinos $\left(\nu_{e}\right)$ over antineutrinos $\left(\bar{\nu}_{e}\right)$, and the two body reactions regulating the neutron to proton ratio $\left(n+\nu_{e} \leftrightarrow p+e^{-}, p+\bar{\nu}_{e} \leftrightarrow n+e^{+}\right)$, drive down the neutron abundance; vice-versa for $\xi_{e}<0$. The effect of a non-zero $\nu_{e}$ asymmetry on the relic abundances of the other light nuclides is much weaker. This is illustrated in Fig. 1, which shows the $\mathrm{D}$ and ${ }^{4} \mathrm{He}$ isoabundance curves in the $\xi_{e}-\eta_{10}$ plane for $N_{\nu}=3$. The nearly horizontal curves reflect the weak dependence of $\mathrm{Y}$ on the baryon density, along with its significant dependence on the neutrino asymmetry. In contrast, the nearly vertical curves reveal the strong dependence of $y_{\mathrm{D}}$ on the baryon density and its weak dependence on any neutrino asymmetry. This complementarity between $y_{\mathrm{D}}$ and $\mathrm{Y}$ permits the pair $\left\{\eta, \Delta N_{\nu}\right\}$ or the pair $\left\{\eta, \xi_{e}\right\}$ to be determined once the primordial abundances of $\mathrm{D}$ and ${ }^{4} \mathrm{He}$ are inferred from the appropriate observational data.

\section{ANALYSIS}

In the "standard" case of $N_{\nu}=3$, the observed abundances of $\mathrm{D}$ and ${ }^{4} \mathrm{He}$ may be used to identify the allowed region in the $\eta-\xi_{e}$ plane. In addition, the cosmic background radiation (CBR) data from WMAP [18], provide complimentary information for the $\eta$ distribution (for different $N_{\nu}$ [15]), which will further restrict the allowed $\eta$ range. In Fig. 2 are shown the $1 \sigma$ and $2 \sigma$ contours in the $\eta_{10}-\xi_{e}$ plane for $N_{\nu}=3$ from a joint CBR (WMAP) - BBN fit using the adopted D and ${ }^{4} \mathrm{He}$ abundances. Although very large when compared to the baryon asymmetry, the allowed values of $\xi_{e}$ are suffi- 
ciently small $\left(\left|\xi_{e}\right| \lesssim 0.1\right)$ that the "extra" energy density contained in such neutrinos is negligible ${ }^{4}$, justifying the claim that these results do, in fact, represent $N_{\nu}=3$.

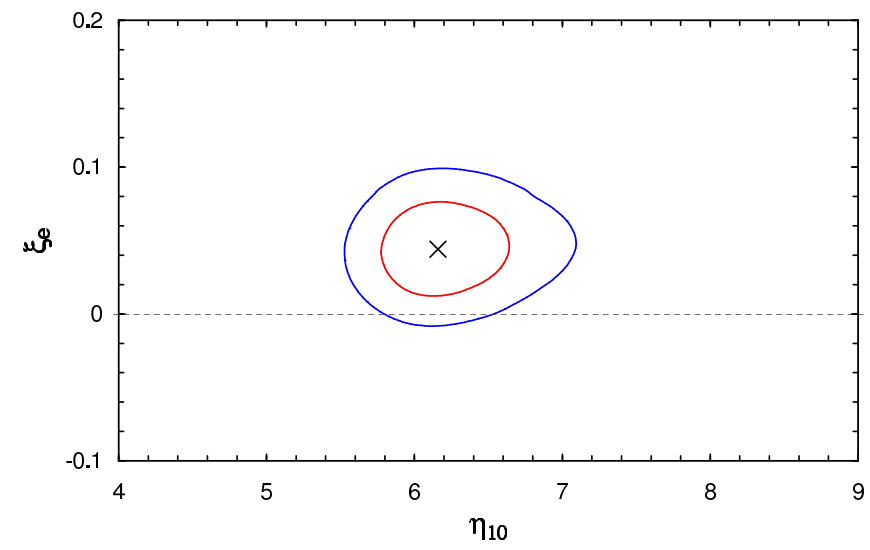

FIG. 2: The $1 \sigma$ and $2 \sigma$ contours in the $\eta_{10}-\xi_{e}$ plane for $N_{\nu}=3$ from a joint CBR - BBN fit using WMAP data and the adopted $\mathrm{D}$ and ${ }^{4} \mathrm{He}$ abundances. The cross marks the best-fit point $\left(\eta_{10}, \xi_{e}\right)=(6.16,0.044)$.

\section{A. The LSND neutrino}

If the LSND results [6] are to be accounted for by the mixing of a "sterile" neutrino with one of the active neutrinos, it is of importance to consider whether the LSND neutrino will be populated, through mixing with the active neutrinos, sufficiently early in the evolution of the universe that it contributes $\Delta N_{\nu}=1$ to the relativistic energy density prior to BBN. Given the parameters required by LSND, in the absence of any significant asymmetry among the active neutrinos the LSND neutrino would, indeed, have come into thermal equilibrium sufficiently early [7]. In this case the new "standard" model would have $N_{\nu}=4$. However, in the absence of a significant neutrino degeneracy, $N_{\nu}=4$ is strongly excluded [15].

In contrast, as the asymmetry in the active neutrinos increases, the sterile-active mixing is delayed [7]. For $\xi_{e} \gtrsim 0.01-0.1$, mixing occurs after the active neutrinos have decoupled from the $e^{ \pm}-\gamma$ plasma. In this case, although the sterile neutrino does still mix with the active ones, the energy is shared among all the neutrinos and $N_{\nu}$ remains equal to 3 . It is interesting, even amusing, that the range of $\xi_{e}$ required by $\mathrm{BBN}$ for $N_{\nu}=3$ is, in fact, in excellent agreement with that required to delay the thermalization of the LSND neutrino $\left(\xi_{e} \gtrsim 0.01-\right.$

\footnotetext{
${ }^{4} \Delta N_{\nu}(\xi)=\sum_{i}\left[\frac{30}{7}\left(\frac{\xi_{i}}{\pi}\right)^{2}+\frac{15}{7}\left(\frac{\xi_{i}}{\pi}\right)^{4}\right] \lesssim 0.013 ;$ see, e.g., [16].
}

0.1 , thereby keeping $N_{\nu} \sim 3$. Thus, the same values of $\xi_{e}$ that reconcile the low value of $\mathrm{Y}$ with the $\mathrm{BBN}$ prediction (for $N_{\nu}=3$ ) also reconcile the LSND sterile neutrino with $\mathrm{BBN}$ by delaying its mixing with the active neutrinos.

\section{B. Arbitrary $N_{\nu}$}

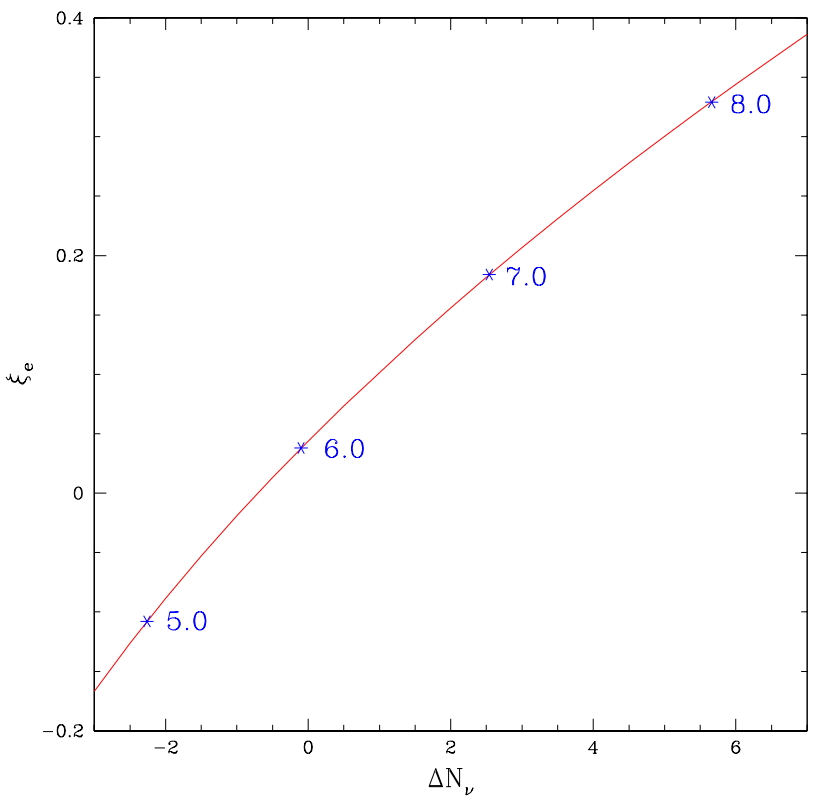

FIG. 3: The approximate $\xi_{e}-\Delta N_{\nu}$ relation corresponding to $y_{\mathrm{D}}=2.6$ and $\mathrm{Y}=0.238$. The numbers shown are the corresponding values of $\eta_{10}$ at those points.

Let us set aside the LSND neutrino, assume that $N_{\nu}=$ 4 , and ask if BBN and the adopted primordial abundances can be reconciled (along with the CBR) through the appropriate choice of a nonzero asymmetry in the active neutrinos.

From BBN with fixed abundances of D and ${ }^{4} \mathrm{He}$, as $N_{\nu}$ increases, so too will the best fit values of $\eta$ and $\xi_{e}$. It is straightforward to understand this effect. As $N_{\nu}$ increases, the early universe expands more rapidly, leaving less time to burn D. As a result, for a fixed baryon to photon ratio, $y_{\mathrm{D}}$ increases with increasing $N_{\nu}{ }^{5}$. To reduce $y_{\mathrm{D}}$ back towards its observed value requires increasing the baryon density. The combination of an increased baryon density and $N_{\nu}=4$, raises the predicted primordial abundance of ${ }^{4} \mathrm{He}$, requiring a larger $\xi_{e}$ to reconcile the BBN

\footnotetext{
${ }^{5}$ This effect on the primordial deuterium abundance is often, but not always [15, 19], ignored. However, for $\left|\Delta N_{\nu}\right| \gtrsim 1$, it is not negligible.
} 
predictions with the data. Indeed, it is clear from Fig. 1 of Kneller et al. [17] that for any (reasonable) choice of $N_{\nu}$ there is a pair of $\left\{\eta_{10}^{*}, \xi_{e}^{*}\right\}$ values for which perfect agreement with the observed D and ${ }^{4} \mathrm{He}$ abundances can be obtained $\left(\chi_{\mathrm{BBN}}^{2}=0\right)$. An approximation to this $\xi_{e}$ $-\Delta N_{\nu}$ relation [20] is shown in Fig. 3 for $y_{\mathrm{D}}=2.6$ and $\mathrm{Y}=0.238$; the numerical values along the curve are the corresponding values of $\eta_{10}$. However, this correlation of increasing $\eta$ with increasing $N_{\nu}$ is broken by the inclusion of WMAP data through the CBR-imposed constraint on the $\eta-N_{\nu}$ relation [15]. As a result, while for $N_{\nu}=4$ the allowed values of $\xi_{e}$ increase from that for $N_{\nu}=3$, the shift in $\eta$ is much more limited. In Fig. 4 is shown the analogue of Fig. 2 (the confidence contours in the $\eta_{10}-\xi_{e}$ plane) for $N_{\nu}=4$.

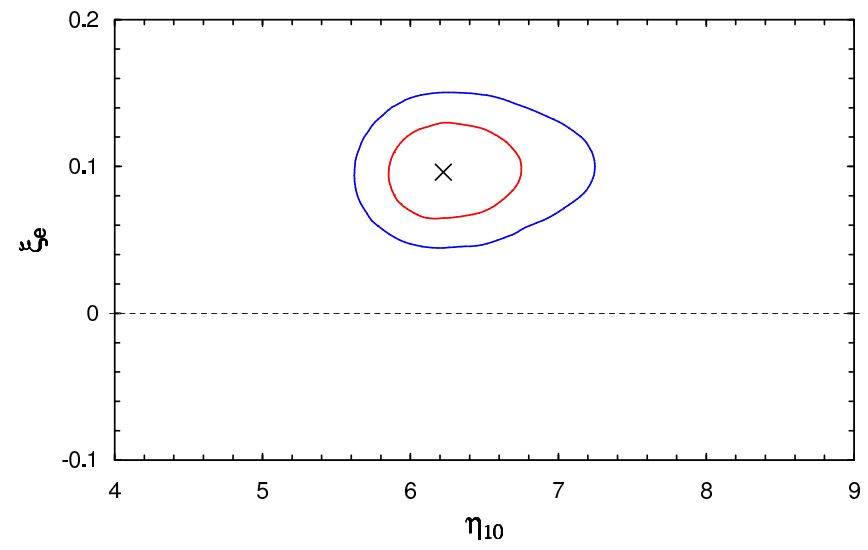

FIG. 4: Same as Fig. 2, except $N_{\nu}=4$. The cross marks the best-fit point $\left(\eta_{10}, \xi_{e}\right)=(6.22,0.096)$.

It is clear that from $\mathrm{BBN}$ alone, reconciling the primordial $\mathrm{D}$ and ${ }^{4} \mathrm{He}$ abundances with the BBN predictions for large $N_{\nu}$ would require too large a baryon to photon ratio to be consistent with the $\eta-N_{\nu}$ constraints from WMAP. The parameter pair $\left\{\Delta N_{\nu}, \xi_{e}\right\}$ can therefore be constrained using the $\left\{\Delta N_{\nu}, \eta\right\}$ likelihood distribution obtained [15] from the WMAP data [18] along with the $\left\{\xi_{e}, \Delta N_{\nu}, \eta\right\}$ likelihood distribution from our BBN analysis, by forming the joint likelihood and marginalizing over $\eta$ with the prior $\pi(\eta)$. Adopting a flat p.d.f. for $\pi(\eta)$ the $\left\{\Delta N_{\nu}, \xi_{e}\right\}$ constraints are derived after calculating (up to a multiplicative constant),

$$
L\left(\xi_{e}, \Delta N_{\nu}\right)=\int L_{\mathrm{BBN}}\left(\xi_{e}, \Delta N_{\nu}, \eta\right) \times L_{\mathrm{WMAP}}\left(\Delta N_{\nu}, \eta\right) d \eta
$$

This analysis results in the allowed regions in the $\Delta N_{\nu}$ $\xi_{e}$ plane shown in Fig. 5, providing limits on the values of $\Delta N_{\nu}$ that can be accommodated by non-zero $\xi_{e}$ without conflicting with the $\eta$ and $\Delta N_{\nu}$ values allowed by WMAP.

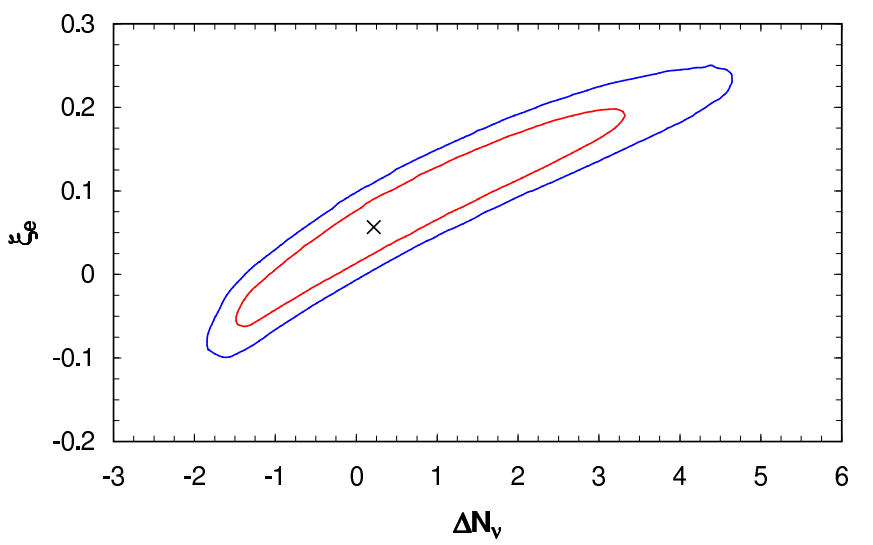

FIG. 5: The $1 \sigma$ and $2 \sigma$ contours in the $\Delta N_{\nu}-\xi_{e}$ plane using WMAP data and BBN with the adopted D and ${ }^{4} \mathrm{He}$ abundances. The cross marks the best-fit point $\left(\Delta N_{\nu}, \xi_{e}\right)=$ $(0.22,0.057)$.

\section{CONCLUSIONS}

Big Bang Nucleosynthesis places strong constraints on any new source of relativistic energy density present during the BBN epoch. Examples include the energy density associated with large asymmetries $\left|\xi_{\mu, \tau}\right|$ between $\mu$ or $\tau$ neutrinos and their antineutrinos, with the right-handed components $\nu_{R}$ of light Dirac neutrinos if they can be efficiently produced by new interactions, or with sterile neutrinos which mix with active neutrinos, as suggested by the LSND experiment. In the absence of a significant neutrino asymmetry the data constrain the equivalent number of new neutrino flavors $\Delta N_{\nu}$ to $\Delta N_{\nu}<0.1-0.3$ (assuming that $\Delta N_{\nu} \geq 0$ ) [15].

BBN also constrains the electron neutrino asymmetry, $\xi_{e}$, which directly affects the neutron to proton ratio and therefore the ${ }^{4} \mathrm{He}$ abundance. For $\Delta N_{\nu}=0$ we find $\xi_{e} \lesssim$ 0.1 , as shown in Fig. 2. However, there remains the possibility of compensation between the effects of $\Delta N_{\nu}$ and $\xi_{e}$, which can relax the constraints on both. The expanded ranges, consistent with WMAP and BBN, are shown in Fig. 5. For $\xi_{e}$ in the range $-0.1 \lesssim \xi_{e} \lesssim 0.3$, values of $\Delta N_{\nu}$ in the range $-2 \lesssim \Delta N_{\nu} \lesssim 5$ are permitted. After marginalizing over $\xi_{e}$, the $2 \sigma$ range for $\Delta N_{\nu}$ (obtained by identifying those values of $\Delta N_{\nu}$ for which the likelihood is above $\left.L_{\max } / e^{2}\right)$ is $-1.7 \lesssim \Delta N_{\nu} \lesssim 4.1$.

The possibility of compensation between a small $\xi_{e}$ and a large $\left|\xi_{\mu, \tau}\right|$ is now excluded given the oscillation parameters inferred for the atmospheric neutrinos and for the LMA solution for the solar neutrinos because of the equilibration of the three $\xi_{i}$ [1]. However, compensation between $\xi_{e}$ and other types of relativistic energy density, such as neutrinos which do not mix with active ones, remains a possibility. We have explored this possibility in detail in this paper finding, for example, that $\Delta N_{\nu}=1$ is consistent with the $\mathrm{BBN}$ and the adopted primordial 
abundances provided that $\xi_{e} \sim 0.1$, as shown in Fig. 4 . Another consequence of our analysis is that even three, light, Dirac neutrinos $\left(\Delta N_{\nu}=3\right)$ can be accomodated provided that $\xi_{e} \sim 0.2$; see Fig. 5 .

Mixing between sterile and active neutrinos must be considered separately, because $\xi_{e}$ can shift the time/temperature at which the sterile neutrinos are produced [7]. Values of $\xi_{e}$ in the range $0.01-0.1$ (which are allowed for $\Delta N_{\nu} \sim 0$; see Fig. 2), are sufficient to keep $N_{\nu}=3$ and thus reconcile the LSND neutrino with BBN.

The compensation between $\Delta N_{\nu}$ and $\xi_{e}$ explored here is, perhaps, fine-tuned. It should not be dismissed, however, because of the significance of these constraints for particle physics (in particular, for models of neutrino mass and mixing), and also because of the importance of $\Delta N_{\nu}$ for the subsequent evolution of structure in the universe, as probed by the $\mathrm{CMB}$ and large scale structure surveys. Also, a large $\xi_{e}$ of the magnitude relevant for $\operatorname{BBN}\left(e . g ., \xi_{e} \sim\right.$ few $\left.\times 10^{-1}\right)$ is huge compared to the baryon asymmetry, so even if it is unlikely, a value in this range would be of profound significance for particle physics and cosmology. While in many models of leptogenesis the asymmetry among the neutral leptons is tied to that in the charged leptons and, hence, is very small $\left(\xi_{e} \approx \eta \sim 10^{-9}\right)$, there are mechanisms for generating the large asymmetry required here [21].

\section{Acknowledgments}

We thank K. Abazajian, G. Fuller and Y. Wong for enlightening discussions. This research was supported by the U.S. DOE under Grants No. DE-FG02-95ER40896, No. DE-FG02-91ER40676, No. DE-FG02-91ER40690, DE-FG02-02ER41216, and No. DOE-EY-76-02-3071, by the NSF under Grant No. PHY99-07949, and by the Wisconsin Alumni Research Foundation. VB, PL, DM and GS thank the Kavli Institute for Theoretical Physics at the University of California, Santa Barbara for its support and hospitality.
[1] C. Lunardini and A. Y. Smirnov, Phys. Rev. D 64, 073006 (2001) [hep-ph/0012056]; A. D. Dolgov, S. H. Hansen, S. Pastor, S. T. Petcov, G. G. Raffelt and D. V. Semikoz, Nucl. Phys. B 632, 363 (2002) [hepph/0201287]; Y. Y. Wong, Phys. Rev. D 66, 025015 (2002) [hep-ph/0203180]; K. N. Abazajian, J. F. Beacom and N. F. Bell, Phys. Rev. D 66, 013008 (2002) [astro-ph/0203442].

[2] K. Eguchi et al. [KamLAND Collaboration], Phys. Rev. Lett. 90, 021802 (2003) [hep-ex/0212021].

[3] See, e.g., P. Langacker, hep-ph/0304053.

[4] K. A. Olive, D. N. Schramm and G. Steigman, Nucl. Phys. B 180, 497 (1981); Phys. Rev. Lett. 43, 239 (1979).

[5] V. Barger, P. Langacker and H. S. Lee, Phys. Rev. D 67, 075009 (2003) [hep-ph/0302066].

[6] A. Aguilar et al. [LSND Collaboration], Phys. Rev. D 64, 112007 (2001) [hep-ex/0104049].

[7] R. Foot and R. R. Volkas, Phys. Rev. Lett. 75, 4350 (1995) [hep-ph/9508275].

[8] E. D. Church, K. Eitel, G. B. Mills and M. Steidl, Phys. Rev. D 66, 013001 (2002) [hep-ex/0203023].

[9] K. Kaneyuki [Super-Kamiokande Collaboration], Nucl. Phys. Proc. Suppl. 112, 24 (2002).

[10] See e.g., V. Barger and D. Marfatia, Phys. Lett. B 555, 144 (2003) [hep-ph/0212126].

[11] A. Bazarko [MiniBooNE Collaboration], Nucl. Phys. Proc. Suppl. 91, 210 (2000) [hep-ex/0009056].
[12] M. Maltoni, T. Schwetz, M. A. Tortola and J. W. Valle, Nucl. Phys. B 643, 321 (2002) [hep-ph/0207157].

[13] H. Pas, L.-g. Song and T. J. Weiler, hep-ph/0209373.

[14] G. Steigman, D. N. Schramm, \& J. E. Gunn, Phys. Lett. 66B, 202 (1977).

[15] V. Barger, J. P. Kneller, H.-S. Lee, D. Marfatia and G. Steigman, hep-ph/0305075.

[16] H. S. Kang \& G. Steigman, Nucl. Phys. B372, 494 (1992).

[17] J. P. Kneller, R. J. Scherrer, G. Steigman and T. P. Walker, Phys. Rev. D64, 123506 (2001).

[18] C. L. Bennett et al., astro-ph/0302207.

[19] R. H. Cyburt, B. D. Fields and K. A. Olive, astroph/0302431.

[20] J. P. Kneller and G. Steigman, "BBN for Pedestrians", in preparation.

[21] P. Langacker, G. Segre and S. Soni, Phys. Rev. D 26, 3425 (1982); I. Affleck and M. Dine, Nucl. Phys. B 249, 361 (1985); J. Liu and G. Segre, Phys. Lett. B 338, 259 (1994); A. Casas, W. Y. Cheng and G. Gelmini, Nucl. Phys. B 538, 297 (1999) [hep-ph/9709289]; J. MarchRussell, H. Murayama and A. Riotto, JHEP 9911, 015 (1999) [hep-ph/9908396]; M. Kawasaki, F. Takahashi and M. Yamaguchi, Phys. Rev. D 66, 043516 (2002) [hepph/0205101]; M. Yamaguchi, [hep-ph/0211163]. 